\title{
Science and Curative Medicine.
}

$\mathrm{I}^{\mathrm{N}}$ $\mathrm{N}$ the fifth Stephen Paget Memorial Lecture before the Research Defence Society (Quart. Jour. Research Defence Soc. summer issue), Dr. H. H. Dale gave some examples of the effect of research on curative medicine. After referring to the founder of the Society, in memory of whom the lecture was instituted, Dr. Dale pointed out the remarkable change which had come over therapeutics in the last thirty years. Not only have new drugs been introduced after experimental trial in the laboratory, but also the investigation of old drugs, found to be of use empirically, has resulted in explanations being obtained for their actions, so that they can be prescribed with confidence that they will be of value, rather than with the pessimism which feels that at any rate they will do no harm. Only passing reference need be made to insulin, salvarsan, ipecacuanha and emetine, as their therapeutic efficacy is so well known: but it might be pointed out that the experimental investigation of emetine led to the differentiation of the different dysenteries, for only one of which emetine is a remedy, and finally to the development of a method of cultivating the amobæ in vitro, so that new substances likely to be of value in amobic dysentery can now be tested first in the test-tube, without the use of experimental animals other than the amœbæ themselves.

Many active remedies are mixtures of different chemical compounds, which it is often impossible to separate by chemical means: even when prepared synthetically in the laboratory, it is not always possible to obtain them in the pure state, free from toxic or inactive by-products: their potency can only be determined by tests on animals or tissues obtained from them. Among these may be mentioned immune sera, the extract of the posterior lobe of the pituitary gland, insulin, salvarsan. Until the potency of pituitary (posterior lobe) extract was controlled under the Therapeutic Substances Act, 1925, one extract might be eighty times stronger than the weakest of a series. Nowadays all are standardised in terms of the International Unit, which is the same amount of activity, whatever preparation is used. Recently a particular make of foreign insulin sold in Great Britain for a short time was imperfectly standardised by animal tests: reports were received that patients relapsed into diabetes because the batch used was too weak, or suffered from dangerous symptoms because another batch was too strong. Animal tests indicated. that the batches might be 40 per cent weaker or 60 per cent stronger than the indications on the labels. The controlling authority under the Therapeutic Substances Act had power to deal with this situation, and no more of such inadequately standardised insulin has been distributed. Insulin would never have come into practical use without control of its activity by regular tests on animals: if such tests were now abandoned, it would lose the greater part of its value in the treatment of diabetes.

In concluding his lecture, Dr. Dale referred to the work of the National Institute for Medical Research in preparing international and British standards for remedies requiring biological test and in developing suitable methods of assay; as well as to the researches being carried out in virus diseases.

\section{Activity Coefficients and the Debye-Hückel Equation.}

$\mathrm{D}^{\mathrm{s}}$ ISCREPANCIES between experimental results and calculations from the Debye-Hückel equation are known to exist even at high dilution with salts of higher valency, as well as for univalent types in non-aqueous solvents. Gronwall, La Mer, and Sandved have assumed that the incomplete mathematical solution of the Poisson-Boltzmann equation as given by Debye and Hückel might explain part at least of the discrepancy. They deduce, as a further approximation, the equation for the activity coefficient of a salt of symmetrical valency type :

$$
\begin{aligned}
\log f= & \frac{-\epsilon^{2} z^{2}}{k T D a} \cdot \frac{1}{2} \cdot \frac{x}{1+x} \\
& +\sum_{m=1}^{\infty}\left(\frac{\epsilon^{2} z^{2}}{k T D a}\right)^{2 m+1}\left[\frac{1}{2} X_{2 m+1}(x)-2 m Y_{2 m+1}(x)\right]
\end{aligned}
$$

in which $\epsilon, z, k, T, D$ have the usual significance in the Debye-Hückel theory; $x=$ ' $a$ ' $k$, where ' $a$ ' is the distance of closest approach of the ions ; and $X(x)$, $Y(x)$ are functions of $x$ defined and tabulated through the fifth approximation, that is $m=2$.

For values of $a / z^{2}>1.5 \mathrm{~A}$., only the first term of this expression, corresponding to the Debye-Hückel approximation, is usually required, but for lower values, corresponding to ' $a$ ' $<6 \mathrm{~A}$. for a bi-bivalent salt, the contribution of higher terms cannot be neglected. In low dielectric solvents the higher terms become important, and may even predominate over the first approximation even for 1,1 valent types. The general solution should yield positive and physically reasonable values for ' $a$ ' which are constant over a legitimate concentration range.

La Mer and Parks, in the June number of the
Journal of the American Chemical Society, describe experiments with cells involving the process $\mathrm{Cd}+\mathrm{PbSO}_{4}$ $=\mathrm{Pb}+\mathrm{CdSO}_{4}$ (using cadmium and lead amalgams), which were in good agreement with the theory for the region 0.0006 to $0.01 \mathrm{molal}$, and yielding positive constant $(3.6 \mathrm{~A}$.) values of ' $a$ ' which are physically plausible. The Debye-Hückel approximation gave impossible values of ' $a$ '. If cadmium sulphate is dissociated incompletely in the classical sense, this influence on the activity coefficient is too small to be detected below 0.01 molal. It is also shown that ' $a$ ' is practically independent of temperature, so that hydration is probably not included in it.

Scatchard, in the same journal, points out that it is incorrect to calculate the heat of dilution from the interionic force formula by differentiation at constant volume, since the experimental data refer to constant pressure. The difference between the two heat quantities is small and can be determined, that between the two dielectric constants is probably also very small, but that between the temperature coefficients at constant pressure and constant volume is generally more important. The celculations are, therefore, made for constant pressure, which involves the addition of a term dependent on the thermal expansion of the solution. The difference is said to amount to about the error of measurement of heat of dilution in very dilute solutions. In the calculation the dielectric constant is assumed independent of concentration, but the extended calculation of Gronwall, La Mer, and Sandved referred to above is applied instead of the simple limiting law of Debye and Hückel. Even in the use of the limiting law, however, neglect of thermal expansion is shown to lead to considerable error.

No. 3229, Vor. 128] 